\title{
GLP-1 secretion by microglial cells and decreased CNS expression in obesity
}

\author{
Camilla Kappe $^{1 \dagger}$, Linda M Tracy ${ }^{2+}$, Cesare Patrone ${ }^{1}$, Kerstin Iverfeldt ${ }^{2^{*}}$ and Åke Sjöholm ${ }^{1^{*}}$
}

\begin{abstract}
Background: Type 2 diabetes (T2D) is a strong risk factor for developing neurodegenerative pathologies. T2D patients have a deficiency in the intestinal incretin hormone GLP-1, which has been shown to exert neuroprotective and anti-inflammatory properties in the brain.

Methods: Here we investigate potential sources of GLP-1 in the CNS and the effect of diabetic conditions on the proglucagon mRNA expression in the CNS. The obese mouse model ob/ob, characterized by its high levels of free fatty acids, and the microglia cell line BV-2 were used as models. mRNA expression and protein secretion were analyzed by qPCR, immunofluorescence and ELISA.

Results: We show evidence for microglia as a central source of GLP-1 secretion. Furthermore, we observed that expression and secretion are stimulated by CAMP and dependent on microglial activation state. We also show that insulin-resistant conditions reduce the central mRNA expression of proglucagon.

Conclusion: The findings that microglial mRNA expression of proglucagon and GLP-1 protein expression are affected by high levels of free fatty acids and that both mRNA expression levels of proglucagon and secretion levels of GLP-1 are affected by inflammatory stimuli could be of pathogenic importance for the premature neurodegeneration and cognitive decline commonly seen in T2D patients, and they may also be harnessed to advantage in therapeutic efforts to prevent or treat such disorders.
\end{abstract}

Keywords: Glucagon-like peptide-1, Microglia, Neuroinflammation, Neuroprotection, Proglucagon

\section{Background}

Type 2 diabetes (T2D) has been identified as a risk factor for developing neurodegenerative pathologies, such as Alzheimer's disease (AD), and stroke [1-3]. Premature cognitive decline is also a common feature in T2D patients. Although it has been speculated that chronic hyperglycemia or recurrent hypoglycemia may be involved in these derangements, the precise nature of the underlying mechanisms remain elusive. T2D patients are not only characterized by hyperglycemia, but typically also have increased circulating levels of free fatty acids that may accumulate in the central nervous system (CNS) [4].

\footnotetext{
* Correspondence: kerstin@neurochem.su.se; ake.sjoholm@ki.se ${ }^{\dagger}$ Equal contributors

2Department of Neurochemistry, Stockholm University, Stockholm 10691, Sweden

${ }^{1}$ Karolinska Institutet, Department of Clinical Science and Education, Södersjukhuset, Stockholm 11883, Sweden
}

Incretin hormones, such as glucagon-like peptide-1 (GLP-1), are derived from proglucagon, which is produced by enteroendocrine L-cells, and augment mealstimulated insulin secretion in a glucose-dependent manner [5]. In T2D, the incretin response has been reported to be impaired as a result of defective GLP-1 secretion [6]. In addition to stimulating insulin secretion, GLP-1 exerts CNS actions such as neuroprotection and reduced appetite $[7,8]$ that appear independent of its glycemic effects. Increasing evidence suggests that GLP-1 and its analogs play a very important neuroprotective role [9]. In GLP-1R knockout mice, neuronal injury is increased after kainate administration [2,10], and GLP-1 has been shown to protect the neuroblastoma cell line SH-SY5Y from amyloid- $\beta$-induced apoptosis [11]. There is also evidence that the GLP-1 mimetic exendin-4, clinically used against T2D, improves cognitive performance [2] and stimulates neurogenesis [12]. In fact, the National Institute on Aging (NIA) is currently

\section{Ciomed Central}


recruiting for a clinical trial with exendin-4 for the treatment of AD. In addition, GLP-1 is well known to have anti-inflammatory properties in different tissues [13]. Considering the importance of GLP-1 in both T2D and neurodegenerative pathologies, and the strong co-morbidity between T2D and different neurodegenerative pathologies, the neuroprotective role and nature of GLP-1 secretion and action in the brain have become hot topics. Further, entirely consistent with an important neuroprotective role of GLP-1, the GLP-1R is abundantly expressed in many parts of the brain, including the hypothalamus, hippocampus and cortex. In contrast, the only known source of GLP-1 secretion in the CNS to date is the nucleus tractus solitarius (NTS) neurons of the brainstem [14], although Iwai et al. reported the presence of GLP-1 immunoreactive material in cultured rat microglia [15]. Here, we aimed to investigate whether there in fact are non-neuronal sources of GLP-1 secretion in the brain, and, if so, how secretion of GLP-1 can be stimulated and how it is affected by obesity and insulin-resistant conditions. Such knowledge may shed light on the pathogenesis of neurodegeneration in T2D patients. Ultimately, this information may also lead to novel ways of stimulating neuroprotection in conditions linked to neurodegeneration, such as T2D, by modulating the secretion of one of the brain's own neuroprotective peptides.

\section{Materials and methods}

\section{Animals}

Obese $(o b / o b)$ and lean $(+/+)$ male mice were obtained from our breeding colony of $\mathrm{C} 57 \mathrm{BL} / 6 \mathrm{~J}-o b /+$ mice. The breeding colony was housed at $23^{\circ} \mathrm{C}$, and the mother was removed from the pups when they were 3 weeks of age. At 3.5 weeks of age, obese and lean pairs were separated from their littermates and were housed individually in solid-bottom, plastic cages with wood shavings and paper as bedding. The experimental procedures were approved by Stockholm South local committee on ethics of animal experiments and performed in accordance with international standards on animal welfare. All mice had food and water available ad libitum. At 6-9 months of age, animals were sacrificed and the brains were removed and cortex and hippocampus dissected. These brain tissues were analyzed together. Alternatively, the brains were used for preparation of tissue sections.

\section{Cell culture and reagents}

The BV-2 cell line [16] was cultured in DMEM Glutamax supplemented with $5 \%$ fetal bovine serum, 10,000 U/ml penicillin and $10 \mathrm{mg} / \mathrm{ml}$ streptomycin sulfate under $5 \%$ $\mathrm{CO}_{2}$ at $37^{\circ} \mathrm{C}$.

Palmitate (sodium palmitate, Sigma-Aldrich, St. Louis, MO) exposure medium was supplemented with $0.5 \%$ bovine serum albumin (BSA, fatty acid free; SigmaAldrich). Palmitate was dissolved in $12.5 \%$ ethanol during heating to $60^{\circ} \mathrm{C}$. Control cells were given vehicle with equal amounts of ethanol as the palmitate-exposed cells. Other reagents used included Forskolin (SigmaAldrich) and lipopolysaccharide (LPS) (Sigma-Aldrich).

Primary rat glial cells cultures consisting of $5-10 \%$ microglia and 90-95\% astrocytes were isolated from the cortex of Sprague-Dawley pups less than $24 \mathrm{~h}$ of age as previously described [17]. Cells were seeded in 60-mm culture dishes and maintained in DMEM Glutamax containing $10 \%$ fetal bovine serum (FBS) and $0.1 \%$ penicillin-streptomycin (PEST) for 20 days (all from GIBCO). Medium was exchanged every 3-4 day.

\section{Preparation of tissue sections}

Isolated mouse brains were placed in $4 \%$ paraformaldehyde (PFA) (Sigma-Aldrich) and fixated overnight at $4^{\circ} \mathrm{C}$. The fixed tissue was then placed in 30\% sucrose and stored in $30 \%$ sucrose at $4^{\circ} \mathrm{C}$. Prior to sectioning the tissue, the stage of the cryocutter was cooled by addition of dry ice. The tissue was mounted on the cold stage using tissue tech (Histolab, Göteborg, Sweden) and covered in dry ice long enough to allow the tissue to take a frozen form. The first few millimeters of tissue was removed, and then $40-\mu \mathrm{m}$-thick coronal sections were cut, using a sliding microtome, throughout the whole brain and placed in antifreeze prior to storage in $-30^{\circ} \mathrm{C}$.

\section{Immunohistochemistry/immunocytochemistry Chromogenic visualization using diaminobenzidine}

Tissue sections were washed in phosphate-buffered saline (PBS) $3 \times 5 \mathrm{~min}$, quenched in hydrogen peroxide for $15 \mathrm{~min}$, before being washed again in phosphate buffer containing $0.25 \%$ Triton $\mathrm{X}-100$ (PBS-T) $3 \times 5 \mathrm{~min}$. Sections were then incubated overnight at $4{ }^{\circ} \mathrm{C}$ with the primary GLP-1-specific antibody (Phoenix peptides) at 1:50 dilution. The primary antibody was detected by use of biotin-conjugated (Vector) secondary antibody (1:200 dilution). Sections were incubated with the secondary antibody for $2 \mathrm{~h}$ at room temperature (approximately $21^{\circ} \mathrm{C}$ ) in phosphate buffer containing $5 \%$ of the appropriate serum and $0.25 \%$ Triton X-100. Following this, a $1-\mathrm{h}$ incubation at room temperature with avidin-biotin complex (ABC kit; Vector) was allowed before subsequent addition of diaminobenzidine.

\section{Fluorescent visualization}

Tissue sections were washed in PBS-T $3 \times 5 \mathrm{~min}$, blocked in $5 \%$ milk and incubated overnight at $4^{\circ} \mathrm{C}$ with primary antibodies (1:50 dilution in PBS-T containing $5 \%$ of the appropriate serum); GLP-1 (Phoenix peptides) and CD11b (AbDSerotec, Raleigh, NC). Sections were washed and incubated at room temperature for $1 \mathrm{~h}$ in 
the dark with the secondary antibodies $(1: 1,000$ and 1:2,000 dilution in PBS-T), ALEXA Fluor 488 donkey anti-rabbit and ALEXA Fluor 568 goat anti-mouse (Invitrogen). The sections were washed again and mounted on glass slides using a soft brush to pick them out of the solution. Sections were allowed to dry in the dark before mounting the cover slip using mounting medium (Histolab).

Primary cells were cultured on a cover glass, washed with pre-warmed PBS-T and fixed with ice-cold 4\% PFA for $10 \mathrm{~min}$ at room temperature. Cells were washed with PBS-T and incubated with primary antibodies (1:50 dilution in PBS-T containing 5\% of the appropriate serum), GLP-1 (Phoenix peptides) and CD11b (AbDSerotec). Cells were washed with $5 \%$ milk solution and incubated at room temperature for $1 \mathrm{~h}$ (kept dark) with the secondary antibodies (1:1,000 and 1:2,000 dilution in PBS-T), ALEXA Fluor 488 donkey anti-rabbit and ALEXA Fluor 568 goat anti-mouse (Invitrogen). The cells were washed again and incubated for $1 \mathrm{~min}$ with DAPI (Invitrogen) prior to a final wash with PBS. The cover slips with cells were then mounted upside down on glass slides using mounting medium (Histolab).

\section{GLP-1 secretion assays}

For palmitate treatment, BV-2 cells were plated at a density of 15,000 cells $/ \mathrm{cm}^{2}$ and grown in 24-well plates for $24 \mathrm{~h}$. Cells were then treated with $0.125 \mathrm{mM}$ palmitate or vehicle for an additional $24 \mathrm{~h}$. Immediately after the $24 \mathrm{~h}$ incubation, medium was collected and DPP-4 inhibitor added $(10 \mu \mathrm{l} / \mathrm{ml})$ (Millipore Corp., Billerica, MA) to prevent GLP-1 degradation. Samples were stored at $-70^{\circ} \mathrm{C}$ pending ELISA.

For acute stimulation with agents, BV-2 cells were plated at a density of 75,000 cells $/ \mathrm{cm}^{2}$ and grown in 24-well plates for $24 \mathrm{~h}$. The medium was discarded, and the cells were washed with pre-warmed Krebs Ringer buffer (KRBH) buffer/0.2\% BSA/0 mM glucose, followed by a 30-min pre-incubation with the same buffer. Cells were then treated with LPS $(1 \mu \mathrm{g} / \mathrm{ml})$ or the adenylyl cyclase activator forskolin $(10 \mu \mathrm{M})$ for $2 \mathrm{~h}$. Immediately thereafter, DPP-4 inhibitor was added $(10 \mu \mathrm{l} / \mathrm{ml})$, and the buffer was collected. Samples were stored at $-70^{\circ} \mathrm{C}$ pending ELISA.

GLP-1 ELISA (Millipore Corp.) was performed according to the manufacturer's instructions. This ELISA is specific for the bioactive forms of GLP-1 [GLP-1 (7-36) amide and GLP-1 (7-37)] and will not detect other forms of GLP-1. There is no crossreactivity with glucagon. All experiments were performed in triplicates and repeated $\geq$ three times to assess consistency of results.

\section{TNFa secretion assay}

BV-2 cells were plated at a density of 15,000 cells $/ \mathrm{cm}^{2}$ and grown for $24 \mathrm{~h}$ prior to exposure to $0.125 \mathrm{mM}$ palmitate for $24 \mathrm{~h}$ or $1 \mu \mathrm{g} / \mathrm{ml}$ LPS for $2 \mathrm{~h}$. The culture medium was collected and analyzed for TNF $\alpha$ on a solid phase ELISA kit (R\&D Systems, Abingdon, UK) according to the manufacturer's protocol.

\section{RNA extraction, CDNA synthesis and quantitative RT-PCR}

BV-2 cells or animal tissue were lysed and RNA extracted using Aurum total RNA mini kit (catalog no. 7326820; BioRad Laboratories) according to the manufacturer's instructions. cDNA was synthesized for qPCR using iScript ${ }^{\mathrm{TM}} \mathrm{cDNA}$ synthesis kit (BioRad Laboratories) according to the manufacturer's instructions.

For qPCR, glyceraldehyde-3-phosphate dehydrogenase (GAPDH) mRNA expression was used as an internal control. GAPDH is one of the most commonly used housekeeping genes for comparisons of gene expression data.

For each sample, the mRNA level of each target gene relative to GAPDH was estimated by calculating the DeltaCt or $\Delta \mathrm{Ct}\left(\mathrm{Ct}_{\text {Target }} \mathrm{Gene}-\mathrm{Ct}_{\mathrm{GAPDH}}\right)$ and then converting to $2^{-\Delta \mathrm{Ct}}$. To compare mRNA levels between experimental groups, the ratio of the average $2^{-\Delta \mathrm{Ct}}$ for each treatment group relative to the control group $\left(2^{-\Delta \Delta C t}\right)$ was determined for each gene. This ratio represents a fold change for each gene. Plotted as arbitrary units are the values generated for fold change. Differences in the scales/size of arbitrary units between the different analyses (see Figure 2) stem from the use of samples from different animals in the different groups skewing the average $\Delta \mathrm{Ct}$ of the groups and thus the fold change $\left(2^{-\Delta \Delta C t}\right)$.

GAPDH primers were designed according to: ATGA CATCAAGAAGGTGGTG, TGTCATACCAGGAAATG AGC using Invitrogen custom primer design software (Invitrogen, Inc.).

Proglucagon primers were designed according to: GATTTTGTGCAGTGGTTGAT, ACTTCTTCTGGGAA GTCTTCG using Invitrogen custom primer design software (Invitrogen, Inc.).

TNF $\alpha$ primers were designed according to: AAAGT CAACCTCCTCTCTGC, GGACTCCGCAAAGTCTAAGT using Invitrogen custom primer design software (Invitrogen, Inc.).

Interleukin- $1 \beta$ primers were designed according to: CTTTTCGTGAATGAGCAGAC, GAGGAAAACACAG GCTCTCT using Invitrogen custom primer design software (Invitrogen, Inc.).

Arginase 1 primers were designed according to: TATGTGTCATTTGGGTGGAT, GCCAATGTACACGA TGTCTT using Invitrogen custom primer design software (Invitrogen, Inc.).

Chitinase-3 primers were designed according to: GACT TGCGTGACTATGAAGC, TGACGGTTCTGAGGAGTA GA using Invitrogen custom primer design software (Invitrogen, Inc.). 
A one-step RT-PCR kit with SYBR Green (iScript ${ }^{\mathrm{TM}}$ one-step RT-PCR kit with SYBR $^{\circledR}$ Green) (BioRad Laboratories) was used for real-time quantitative RT-PCR. This kit utilizes iScript RNase $\mathrm{H}+$ reverse transcriptase and hot-start iTaq DNA polymerase. GAPDH was used as a housekeeping gene for normalization.

\section{Statistical analysis}

Comparisons between control and single treatment groups with normalized distribution of data were done using two-tailed Student's $t$ test. In the case that normality tests failed, Mann-Whitney rank sum test was used for comparisons between the control and single treatment group. Correlations were evaluated by determining the Pearson correlation coefficients. $P<0.05$ was deemed statistically significant.

\section{Results}

GLP-1-positive cells are detected in cerebral cortex of mice and significantly reduced in an obese insulin-resistant mouse model

To achieve the aim of this study, we investigated whether GLP-1-positive cells could be detected in parts of the brain outside of the nucleus of the solitary tract, where proglucagon mRNA expression has been previously observed. Results show that GLP-1-positive cells were indeed detected in cortex and hippocampus of adult C57 black and ob/ob male mice (Figure 1A). In addition, the number of GLP-1-positive cells was significantly reduced in the obese insulin-resistant ob/ob mouse model as compared to normal C57 black mice (Figure 1B).
Proglucagon mRNA expression is detected in cerebral cortex and hippocampus of mice and significantly reduced in an obese insulin-resistant mouse model, while also strongly correlated to the mRNA expression of the proinflammatory tumor necrosis factor-a (TNFa)

To further confirm our finding of GLP-1-positive cells in an area outside of the nucleus of the solitary tract, where proglucagon mRNA expression has been previously observed, we investigated if proglucagon gene expression could be detected. Results show that proglucagon mRNA expression was indeed detected in the cortex and hippocampus of adult C57 black and ob/ob male mice (Figure 2A). In addition, the expression of the proglucagon gene was significantly reduced in the obese insulin-resistant $o b / o b$ mouse model as compared to normal C57 black mice (Figure 2A). Since low-grade systemic inflammation is recognized in T2D [18], we also investigated if the expression of the proglucagon gene was correlated to the presence of inflammation in these areas of the brain. Thus, we determined the mRNA expression of the inflammatory markers TNF $\alpha$ and interleukin-1 $\beta$ (IL-1 $\beta)$ in the same tissue samples. Results show that, whereas no significant difference in the mRNA expression of IL-1 $\beta$ (115 $\pm 15 \%$ of expression in C57 black) could be detected between C57 black and ob/ob mice, significantly lower levels of TNF $\alpha$ mRNA expression were detected in $o b / o b$ mice (Figure 2B). Further, there was a strong positive correlation between proglucagon mRNA expression and the mRNA expression of TNF $\alpha$ (Figure 2C).

Microglial cells express the proglucagon gene and secrete GLP-1 in a CAMP-dependent manner

Since neurons in cerebral cortex and hippocampus have been reported to lack expression of proglucagon [19],
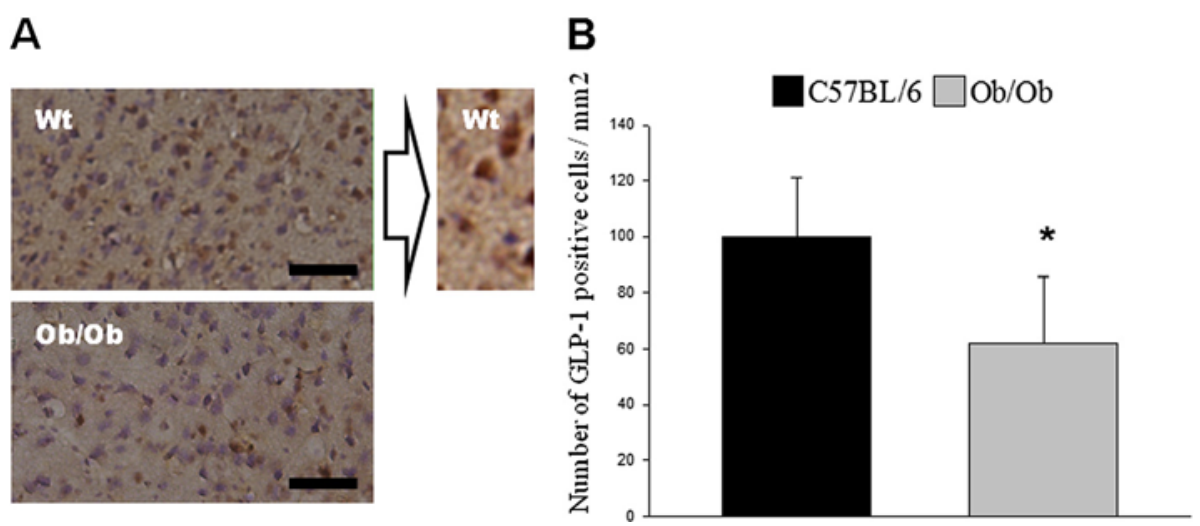

Figure 1 The number of GLP-1-positive cells in the frontal cortex is reduced in an obese insulin-resistant mouse model. A.

Representative images from the frontal cortex adjacent to the hippocampus of 36-week-old mice showing relative abundance of GLP-1-positive cells (brown) in C57 black control animals (top left panel) —increased magnification (100 X) of GLP-1-positive cells (top right panel) —as compared to obese insulin-resistant ob/ob mice (bottom panel). Scale bar: $80 \mu \mathrm{m}$. B. Statistical analysis showing a significant decrease in the number of GLP-1-positive cells/area_-in the frontal cortex adjacent to the hippocampus -in obese insulin-resistant ob/ob mice as compared to healthy controls. Data from 4 C57 black mice and 4 ob/ob mice. Bars represent mean \pm SEM. ${ }^{*} P<0.05$ for a chance difference vs. C57 black mice. Statistical analysis was performed using Mann-Whitney rank sum test. 

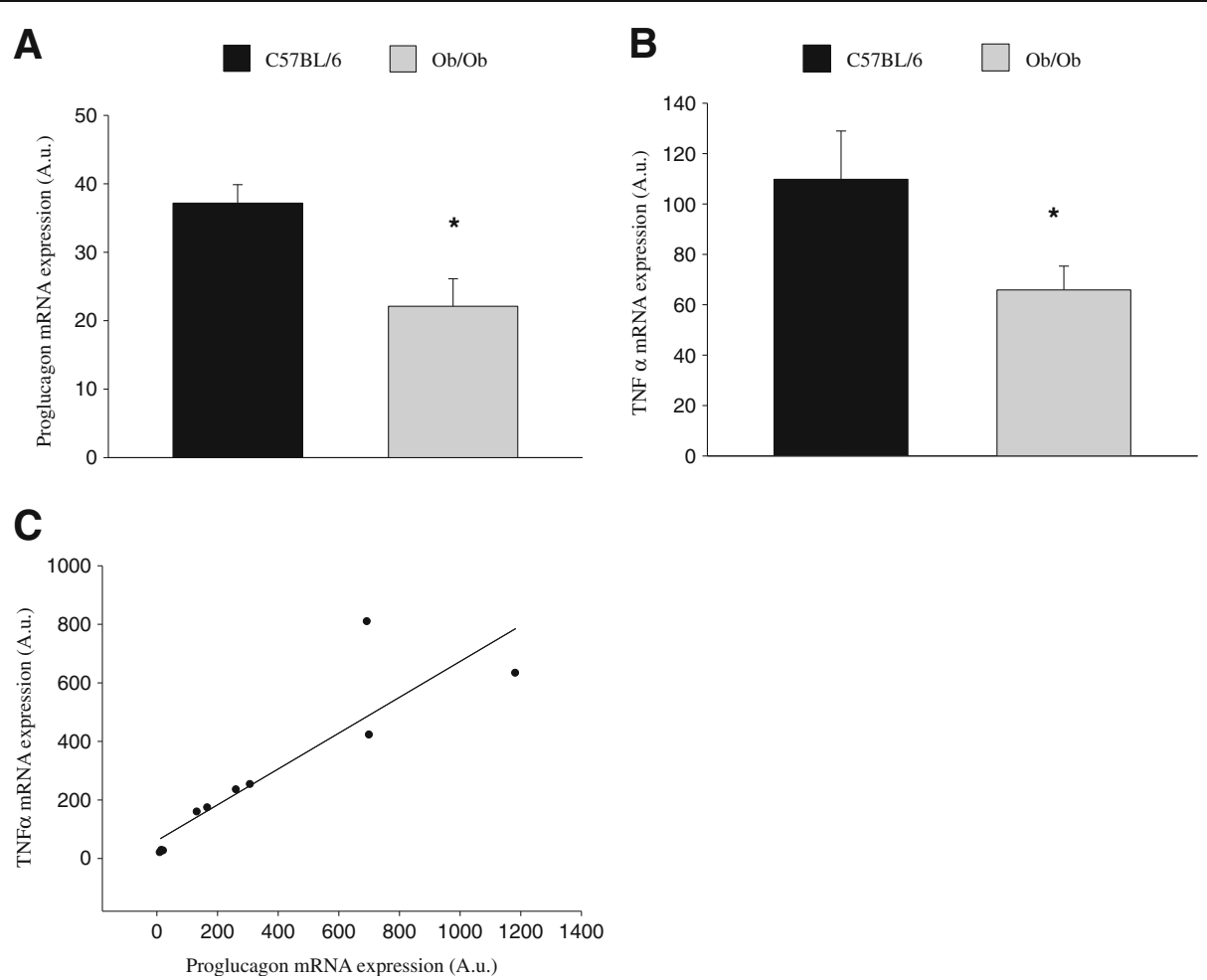

Figure 2 CNS proglucagon mRNA expression in the frontal cortex is reduced in an obese insulin-resistant mouse model. A. Proglucagon mRNA expression in obese ob/ob mice as compared to C57 black littermates. Data are from two groups of adult mice, in total 6 C57 black mice and 9 ob/ob mice, 6-9 months of age. B. TNFa mRNA expression in obese ob/ob mice as compared to C57 black littermates. Data from the same animals as in A. Proglucagon and TNFa mRNA expression was normalized with mRNA expression of GAPDH, which was used as an internal control. Bars represent mean \pm SEM. ${ }^{*} P<0.05$ for a chance difference. Statistical analysis was performed using Mann-Whitney rank sum test. C. Correlation between proglucagon mRNA and TNFa mRNA. Data from three C57 black mice and five ob/ob mice, 6 months of age; qPCR run in duplicate at two independent occasions. A.u.; arbitrary units.

and after observing the strong correlation of proglucagon mRNA expression in these areas to the expression of the inflammatory marker TNF $\alpha$, we investigated resident immune cells of the CNS (microglia) as a possible source of the proglucagon mRNA expression observed in these areas of the brain. Co-staining of tissue sections from the cortex for the CD11b antigen expressed on monocytes/macrophages and microglia, and GLP-1, revealed GLP-1-positive microglia in the murine cortex (Figure 3A). We also investigated GLP-1 expression in primary rat mixed glial cells and detected GLP-1positive microglia cells (Figure $3 \mathrm{~B}$ ). To further confirm microglia as a source of proglucagon mRNA expression, as well as regulated GLP-1 secretion, we used the murine microglia BV-2 cell line. We found that these microglia-derived cells indeed express the proglucagon gene and secrete GLP-1 (Figure 3C,D). Previously, cyclic AMP has been shown to stimulate proglucagon gene transcription and GLP-1 production in other cell types [20]. As expected, in microglial cells both mRNA expression of the proglucagon gene and GLP-1 secretion were enhanced by adenylyl cyclase activation induced by forskolin (Figure 3C,D).

\section{GLP-1 secretion from microglial cells is regulated by inflammation}

In agreement with previous studies [21], classical activation of BV-2 microglial cells with lipopolysaccharide (LPS) increases the secretion of TNF $\alpha$ (Figure 4A). We report here that acute $(2 \mathrm{~h})$ activation of BV-2 cells with LPS also transiently increases proglucagon mRNA expression, while there is a clear trend toward reduced proglucagon expression after 24 h (Figure 4B). However, the increased mRNA expression after $2 \mathrm{~h}$ occurs in conjunction with a significant decrease in the amount of GLP-1 secreted from these cells (Figure 4C).

\section{Proglucagon mRNA expression is decreased in} alternatively activated microglial cells in an in vitro model of T2D

Since proglucagon mRNA expression was reduced in the cortex and hippocampus of $o b / o b$ mice (Figure 1A), we 
A

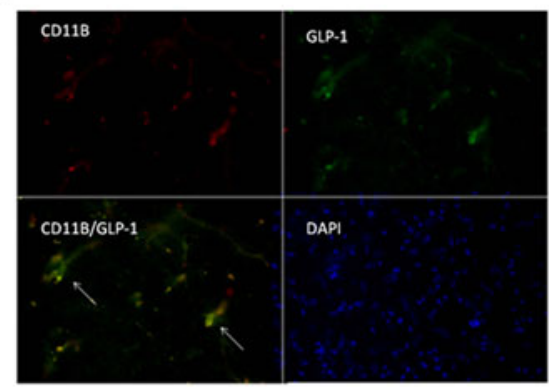

C Control $\square 10 \mu \mathrm{M}$ Forskolin

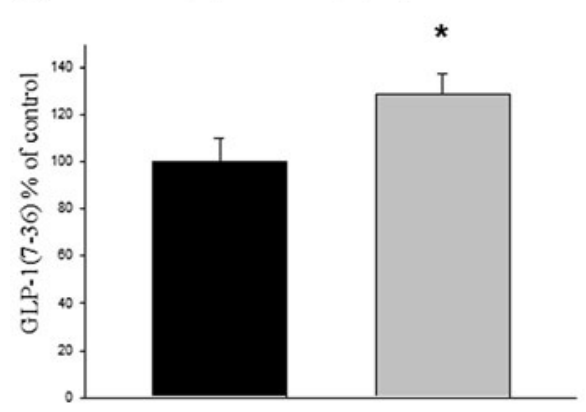

B
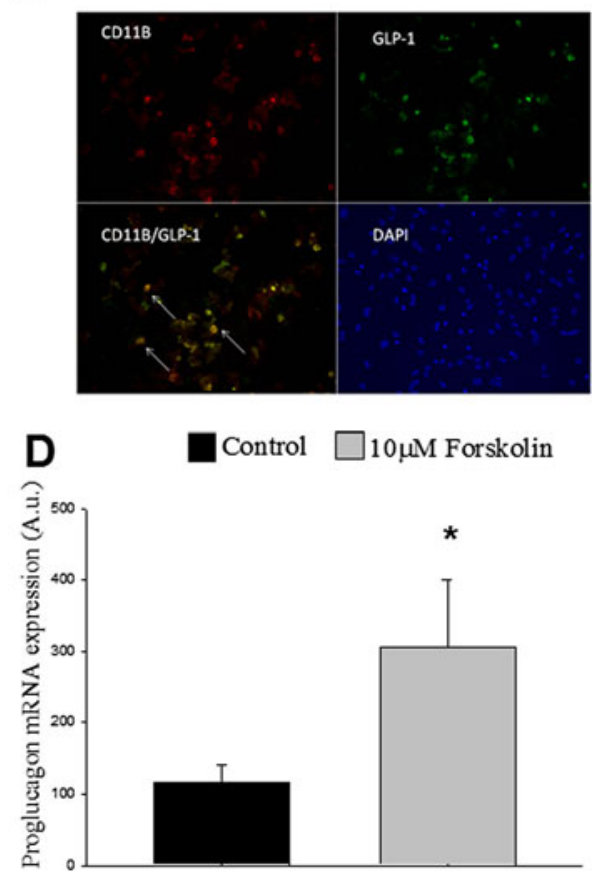

Figure 3 Microglia express proglucagon mRNA and express and secrete GLP-1. GLP-1-positive cells in A, tissue sections from the murine cortex, and B, primary glial cell cultures, GLP-1-positive (green, top right panel) microglia (red, top left panel). Cells positive for GLP-1 are also positive for the microglia marker CD11b (bottom left panel), whereas the remaining glial cells in the culture (indicated by blue DAPI staining in the bottom right panel) are positive for neither GLP-1 nor CD11b. White arrows indicate cells double-positive for CD11b and GLP-1. C. BV-2 cells secrete GLP-1, and this secretion is stimulated in response to a 2-h treatment with $10 \mu \mathrm{M}$ of the adenylyl cyclase activator forskolin. GLP-1 secretion was measured as pM and presented as percent of control. D. Expression of proglucagon is increased in response to a 2-h treatment with $10 \mu \mathrm{M}$ forskolin. Proglucagon mRNA expression was normalized to mRNA expression of GAPDH, which was used as an internal control. Bars represent mean \pm SEM, $n=3-4$, in triplicates. ${ }^{*} P<0.05$ for a chance difference $v$ s. untreated controls. Statistical analysis was performed using Student's t-test.

aimed to determine the effects of diabetic conditions on GLP-1 secretion from microglial cells in vitro. To simulate diabetic conditions, we cultured the BV-2 microglial cells in the presence or absence of a high concentration $(0.125 \mathrm{mM})$ of the saturated fatty acid palmitate and determined the amount of GLP-1 secreted into the medium, as well as proglucagon mRNA expression levels. Our results demonstrate that high levels of fatty acid significantly reduced proglucagon mRNA expression after $2 \mathrm{~h}$, as well as after $24 \mathrm{~h}$. However, no significant effect on GLP-1 secretion could be detected in response to simulated hyperlipidemia (24-h incubation with palmitate) (Figure 5A,B). Since a strong positive correlation between proglucagon and TNF $\alpha$ mRNA expression was detected in vivo (Figure 1C), the effect of high levels of palmitate on TNF $\alpha$ mRNA expression and secretion from the microglial cells was determined. In line with the in vivo results, palmitate significantly reduced TNF $\alpha$ secretion and mRNA expression after $24 \mathrm{~h}$ (Figure 5C,D). To verify that these effects were not the result of a toxic effect of palmitate, we measured protein content in the wells at the end of the 24-h incubation.
Our data demonstrate that palmitate does not alter the amount of protein in the wells after $24 \mathrm{~h}$ (control: $3.90 \pm$ $0.29 \mu \mathrm{g} / \mu \mathrm{l} v s$. palmitate: $3.65 \pm 0.35 \mu \mathrm{g} / \mu \mathrm{l})$. The decrease in TNF $\alpha$ mRNA expression and secretion in response to simulated hyperlipidemia $(24 \mathrm{~h}$ incubation with palmitate) is unexpected, considering the systemic inflammation in T2D and that TNF $\alpha$ secretion increases in response to a classical activation of microglia cells. This prompted us to investigate a possible alternative activation of the microglial cells in response to palmitate. As shown in Figure 5E, we found that hyperlipidemia simulated by a 24-h in vitro incubation with $0.125 \mathrm{mM}$ palmitate significantly increased the mRNA expression of both chitinase 3-like protein 3 ( $\mathrm{Ym} 1$ ) and arginase 1 -two markers of alternative activation of immune cells [22], while LPS, known to induce classical activation of microglia, significantly reduced the expression of arginase 1 after $24 \mathrm{~h}$ (Figure 5F). No significant effect on the expression of arginase 1 could be detected after $2 \mathrm{~h}$ with LPS/palmitate (LPS: $103.7 \pm 13.7$ vs. LPS control: $106.7 \pm 28.3 /$ palmitate: $72 \pm 11.6$ vs. palmitate control $116.9 \pm 30.6$ ). 

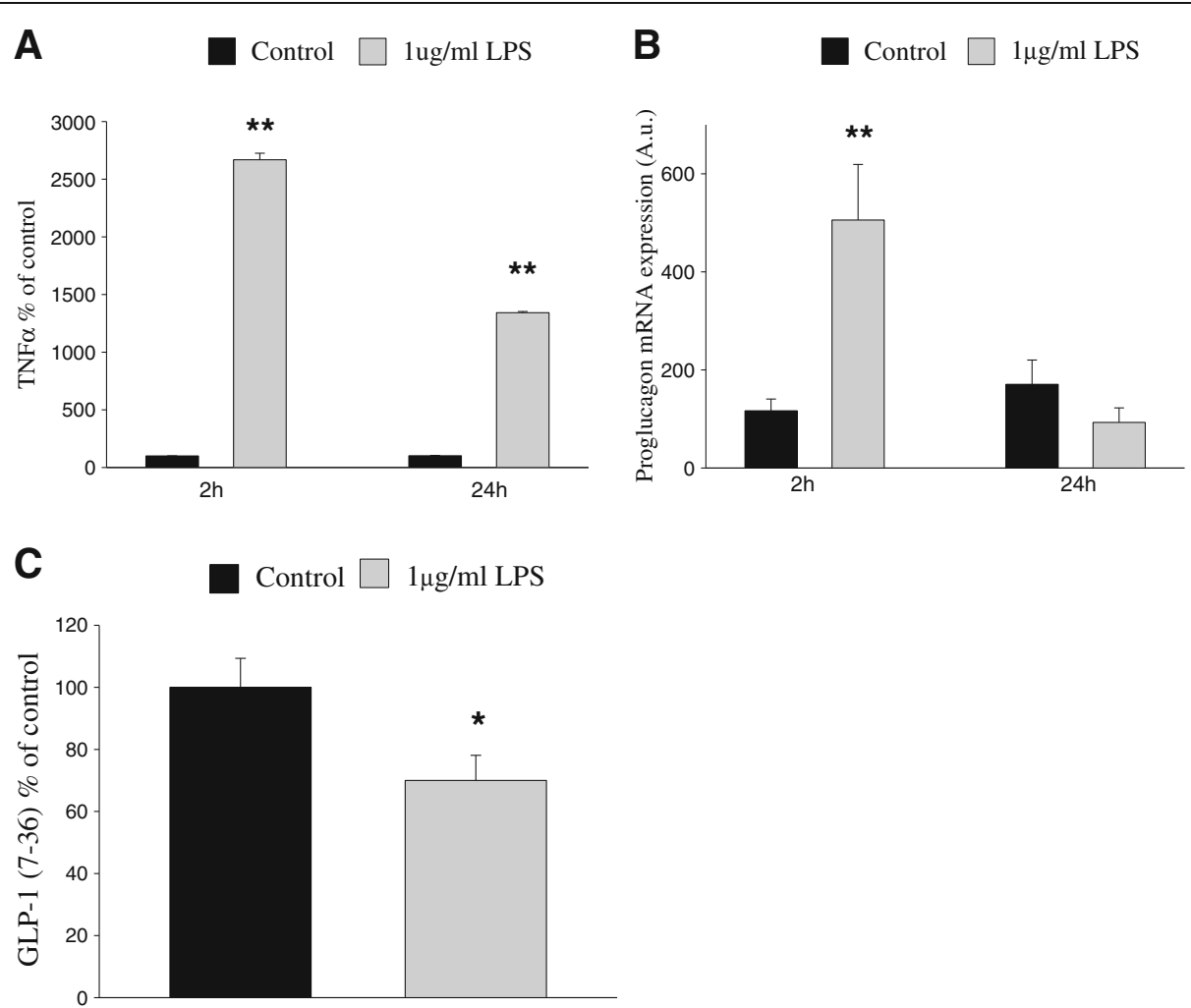

Figure 4 Microglial GLP-1 secretion is decreased in response to inflammatory stimuli. A. TNFa secretion is increased in response to $2-h$ and 24-h LPS treatment. TNFa secretion was measured as $\mathrm{pg} / \mathrm{ml}$ and presented as percent of control. B. Expression of proglucagon is transiently increased in response to a $2-h$ treatment with LPS, with a clear trend toward reduced levels after 24 h. Proglucagon mRNA expression was normalized to mRNA expression of GAPDH, which was used as an internal control. C. Secretion of GLP-1 from BV-2 cells is inhibited by a 2-h treatment with $1 \mu \mathrm{g} / \mathrm{ml}$ LPS. GLP-1 secretion was measured as PM and presented as percent of control. Bars represent mean \pm SEM, $n=3-4$, in triplicates. ${ }^{*} P<0.05$ for a chance difference vs. untreated controls. Statistical analysis was performed using Mann-Whitney rank sum test $(\mathbf{A}, \mathbf{B})$ and Student's t-test $(\mathbf{C})$.

\section{Discussion}

Here we show for the first time that BV-2 microglial cells express the proglucagon gene and secrete GLP-1, processes that are both enhanced by cAMP. We also show that this expression of proglucagon is downregulated, in conjunction with a reduced number of GLP-1positive cells, in obese insulin-resistant animals in vivo, an effect mimicked in vitro in a diabetic milieu characterized by high levels of fatty acids. In line with our central finding that microglial cells secrete GLP-1, we also demonstrate that proglucagon mRNA expression and GLP-1-positive cells are detected in areas of the brain distinct from the brainstem and the NTS neuronswhere proglucagon expressing neurons have previously been identified.

Throughout this study we have used $o b / o b$ mice, a well-known and widely used animal model for obesity and insulin resistance. The $o b / o b$ mice are characterized by high levels of free fatty acids and systemic inflammation [23-25]. The BV-2 cell line is commonly used since it offers the advantage of being comprised exclusively of microglial cells and at the same time retains a highly similar response pattern to primary microglia, especially in response to inflammatory stimuli [16].

We report here that acute adenylyl cyclase and PKA activation by forskolin increases proglucagon mRNA expression and GLP-1 secretion from microglia cells, showing that the proglucagon and GLP-1 levels can be manipulated by different stimuli. Further, we report that classical activation of these cells in vitro, induced by acute LPS treatment, reduces GLP-1 secretion, while acutely and transiently increasing proglucagon mRNA expression, indicating that the acute effect of LPS treatment initially interferes with the secretory mechanism. The specific steps impaired by LPS will be investigated in forthcoming studies.

Due to their insulin resistance, T2D patients often have elevated levels of plasma FFAs [26,27], and recent studies indicate an increased accumulation of fatty acids also in the CNS in diabetes [4]. Consequently, we aimed to evaluate whether high concentrations of fatty acids would alter the activation state and/or the proglucagon 


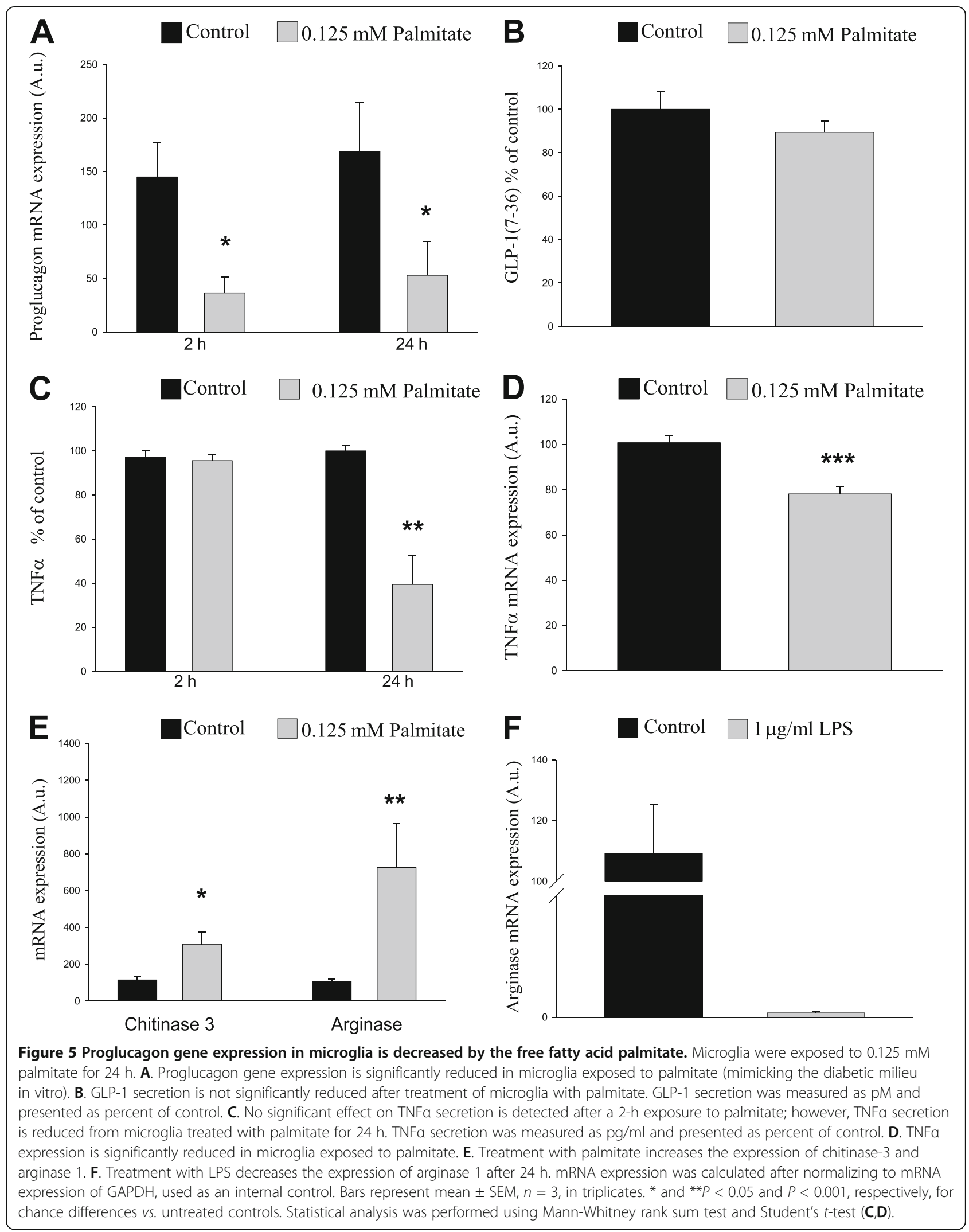


mRNA expression of microglial cells. Palmitate was chosen to evaluate the effect of high concentrations of FFAs relevant to T2D, as it is the most studied FFA in endocrine cell types. We used complexes where the molar ratio of FFA to albumin was 2:1, which is physiologically relevant. We report here that diabetic conditions simulated by palmitate treatment in vitro significantly reduce proglucagon mRNA expression in microglial cells.

In support of microglia being a source of the proglucagon mRNA expression detected in C57 black mice, the inhibitory effect of high levels of palmitate on proglucagon mRNA expression from microglial cells is in line with the downregulation of proglucagon gene expression in the $o b /$ $o b$ mice. However, GLP-1-positive cells are reduced in $o b /$ $o b$ mice, while GLP-1 secretion is not modified in palmitate-treated microglial cells. This discrepancy could stem from the relatively short treatment $(24 \mathrm{~h})$ with palmitate in vitro. It is possible that reduced GLP-1 secretion may come as a consequence of reduced proglucagon expression and be detectable at a later time point. Another possibility is that direct effects on GLP-1 secretion by the relatively short treatment with palmitate in vitro compensates for a reduced number of GLP-1-positive cells in the culture. Future studies will aim to investigate the relevance of these hypotheses. Noticeably, our results demonstrate that LPS and 24-h treatment of BV-2 cells with the saturated fatty acid palmitate will induce opposite effects, where palmitate treatment will reduce the mRNA expression and secretion of TNF $\alpha$, whose induction is characteristic of a classical activation (LPS activation) of these immune competent cells, while inducing the alternative form of microglia activation. Differential activation in response to fatty acids and LPS has been reported for macrophages in other studies [28]. However, there are also studies supporting an increase in the mRNA expression and secretion of TNF $\alpha$ from monocytes in response to palmitate, although these studies generally use much higher concentrations of palmitate $(0.5 \mathrm{mM})$ [29], which may not have the same clinical relevance as the concentration used in this study. Little et al. observed an increase of IL-8 and MCP-1 in palmitate-treated THP-1 cells [30], using the same concentration $(0.125 \mathrm{mM})$ of palmitate as the present study. However, the cell type and the time differ from this study. Further, it is somewhat inconclusive what the elevated levels of IL- 8 indicate since IL- 8 has been reported to be associated with M2c (a subtype of the M2 phenotype)-activated macrophages, as reviewed by David and Kroner [31], as well as with the M1 phenotype. Moreover, it is well known that the microenvironment and previous insults to the cells can contribute to the activation state, thus making it difficult to pinpoint the exact effect of different stimuli. Further, our in vivo data showing reduced TNF $\alpha$ mRNA expression in an obese insulin- resistant mouse model further support the fact that high levels of fatty acids will reduce TNF $\alpha$ mRNA expression and secretion.

The positive correlation between TNF $\alpha$ and proglucagon mRNA expression in C57 black and ob/ob mice identified in this study, for example, decreased mRNA expression of an inflammatory cytokine and simultaneous decreased mRNA expression of a neuroprotective peptide, may have its biological importance in protecting the neurons from cytotoxic factors secreted to kill off invading pathogens. This is of course very speculative, but the possible biological relevance and importance of these novel findings should lead to a thorough assessment of the mechanisms.

The decrease of TNF $\alpha$ in $o b / o b$ mice could stem from a change in microglia induced by fatty acids toward more alternatively activated microglia, as indicated by the present study, and thus lower levels of TNF $\alpha$. However, this study does not investigate the effects of simulated hyperlipidemia in the context of LPS activation of microglia. It is possible, as indicated by studies in other tissues [32], that, in response to invading pathogens/infections and release of endotoxins, the high levels of fatty acids contribute to an enhanced/prolonged immune response that may have cytotoxic effects. To further investigate proglucagon mRNA expression and GLP-1 secretion in response to LPS/amyloid- $\beta$ under hyperlipidemic conditions remains part of our future studies. In summary, this study provides novel information on GLP-1 production in the brain. We demonstrate that NTS neurons are not the only central source of GLP-1. Proglucagon is expressed in other areas of the brain and significantly reduced by (pre) diabetic conditions in vivo and in vitro. Specifically, this study demonstrates for the first time that microgliaderived cells secrete GLP-1 and that this secretion is regulated by the activation state of these cells, by forskolin leading to PKA stimulation and by LPS. One of our future research goals, in addition to further characterizing the mechanisms that regulate GLP-1 secretion from microglia, is to determine the effects of insulin-resistant conditions on GLP-1 secretion in vivo by comparing nondiabetic $\mathrm{C} 57$ black and obese $o b / o b$ mice.

The novel information provided in this study and the central finding that microglia secrete the neuroprotective peptide GLP-1 may have very interesting pathogenic and therapeutic implications for the diabetes/neurodegenerative disease interface. Modulating the activation state of-and the GLP-1 secretion from-microglia may be a novel option for treating neurodegenerative diseases.

\section{Abbreviations}

TNFa: Tumor necrosis factor a; GLP-1: Glucagon-like peptide-1; PKA: Protein kinase A; LPS: Lipopolysaccharide; T2D: Type 2 diabetes; Ym1: chitinase 3-like 3. 


\section{Competing interests}

The authors declare that they have no competing interests.

\section{Authors' contributions}

CK and LMT: Conceived the research plan, performed and designed the experiments, contributed to discussions, wrote the manuscript, performed analysis of data, and acquired and processed images and figures. CP: Participated in discussions and manuscript preparation and contributed to the research plan. Kl: Provided expertise on AD and neuroinflammation, participated in manuscript preparation and discussions, and contributed to the research plan. Ås: Provided expertise in T2D, participated in the manuscript preparation and discussions, provided the animal models used and contributed to the research plan. All authors read and approved the final manuscript.

\section{Acknowledgement}

The authors thank Petra Wolbert, Richelle Fall and Diana Rydholm for maintaining the mouse colony and genotyping. This work was supported by the Diabetes Research \& Wellness Foundation, the Regional Agreement on Medical Training and Clinical Research (ALF) between Stockholm County Council and the Karolinska Institutet, the AFA Insurance, Gun and Bertil Stohnes Foundation, and the Swedish Dementia Foundation.

Received: 24 October 2012 Accepted: 29 November 2012 Published: 23 December 2012

\section{References}

1. Giorda CB, Avogaro A, Maggini M, Lombardo F, Mannucci E, Turco S, Alegiani SS, Raschetti R, Velussi M, Ferrannini E: Incidence and risk factors for stroke in type 2 diabetic patients: the DAl study. Stroke 2007, 38:1154-1160.

2. Isacson R, Nielsen E, Dannaeus K, Bertilsson G, Patrone C, Zachrisson O, Wikstrom $L$ : The glucagon-like peptide 1 receptor agonist exendin-4 improves reference memory performance and decreases immobility in the forced swim test. Eur J Pharmacol 2011, 650:249-255.

3. Kroner Z: The relationship between Alzheimer's disease and diabetes: Type 3 diabetes? Altern Med Rev 2009, 14:373-379.

4. Karmi A, lozzo P, Viljanen A, Hirvonen J, Fielding BA, Virtanen K, Oikonen V, Kemppainen J, Viljanen T, Guiducci L, et al: Increased brain fatty acid uptake in metabolic syndrome. Diabetes 2010, 59:2171-2177.

5. Drucker DJ: The role of gut hormones in glucose homeostasis. J Clin Invest 2007, 117:24-32

6. Laakso M, Zilinskaite J, Hansen T, Boesgaard TW, Vanttinen M, Stancakova A, Jansson PA, Pellme F, Holst JJ, Kuulasmaa T, et al: Insulin sensitivity, insulin release and glucagon-like peptide-1 levels in persons with impaired fasting glucose and/or impaired glucose tolerance in the EUGENE2 study. Diabetologia 2008, 51:502-511.

7. Williams DL: Minireview: finding the sweet spot: peripheral versus central glucagon-like peptide 1 action in feeding and glucose homeostasis. Endocrinology 2009, 150:2997-3001.

8. Perry T, Holloway HW, Weerasuriya A, Mouton PR, Duffy K, Mattison JA, Greig NH: Evidence of GLP-1-mediated neuroprotection in an animal model of pyridoxine-induced peripheral sensory neuropathy. Exp Neurol 2007, 203:293-301

9. Darsalia V, Mansouri S, Ortsater H, Olverling A, Nozadze N, Kappe C, Iverfeldt K, Tracy LM, Grankvist N, Sjoholm A, Patrone C: Glucagon-like peptide-1 receptor activation reduces ischemic brain damage following stroke in type 2 diabetic rats. Clin Sci (Lond) 2012, 122:473-483.

10. During MJ, Cao L, Zuzga DS, Francis JS, Fitzsimons HL, Jiao X, Bland RJ, Klugmann M, Banks WA, Drucker DJ, Haile CN: Glucagon-like peptide-1 receptor is involved in learning and neuroprotection. Nat Med 2003, 9:1173-1179.

11. Li Y, Tweedie D, Mattson MP, Holloway HW, Greig NH: Enhancing the GLP1 receptor signaling pathway leads to proliferation and neuroprotection in human neuroblastoma cells. J Neurochem 2010, 113:1621-1631.

12. Bertilsson G, Patrone C, Zachrisson O, Andersson A, Dannaeus K, Heidrich J, Kortesmaa J, Mercer A, Nielsen E, Ronnholm H, Wikstrom L: Peptide hormone exendin-4 stimulates subventricular zone neurogenesis in the adult rodent brain and induces recovery in an animal model of Parkinson's disease. J Neurosci Res 2008, 86:326-338.
13. Li Y, Duffy KB, Ottinger MA, Ray B, Bailey JA, Holloway HW, Tweedie D, Perry $T$, Mattson MP, Kapogiannis D, et al: GLP-1 receptor stimulation reduces amyloid-beta peptide accumulation and cytotoxicity in cellular and animal models of Alzheimer's disease. J Alzheimers Dis 2010, 19:1205-1219.

14. Vrang N, Hansen M, Larsen PJ, Tang-Christensen M: Characterization of brainstem preproglucagon projections to the paraventricular and dorsomedial hypothalamic nuclei. Brain Res 2007, 1149:118-126.

15. Iwai T, Ito S, Tanimitsu K, Udagawa S, Oka J: Glucagon-like peptide-1 inhibits LPS-induced IL-1beta production in cultured rat astrocytes. Neurosci Res 2006, 55:352-360.

16. Henn A, Lund S, Hedtjarn M, Schrattenholz A, Porzgen P, Leist M: The suitability of BV2 cells as alternative model system for primary microglia cultures or for animal experiments examining brain inflammation. ALTEX 2009, 26:83-94.

17. Ramberg $V$, Tracy LM, Samuelsson M, Nilsson LN, Iverfeldt K: The CCAAT/ enhancer binding protein (C/EBP) delta is differently regulated by fibrillar and oligomeric forms of the Alzheimer amyloid-beta peptide. J Neuroinflammation 2011, 8:34.

18. Sjoholm A, Nystrom T: Endothelial inflammation in insulin resistance. Lancet 2005, 365:610-612.

19. Merchenthaler I, Lane M, Shughrue P: Distribution of pre-pro-glucagon and glucagon-like peptide-1 receptor messenger RNAs in the rat central nervous system. J Comp Neurol 1999, 403:261-280.

20. Brubaker PL, Schloos J, Drucker DJ: Regulation of glucagon-like peptide-1 synthesis and secretion in the GLUTag enteroendocrine cell line. Endocrinology 1998, 139:4108-4114.

21. Persson M, Brantefjord M, Hansson E, Ronnback L: Lipopolysaccharide increases microglial GLT-1 expression and glutamate uptake capacity in vitro by a mechanism dependent on TNF-alpha. Glia 2005, 51:111-120.

22. Colton CA, Mott RT, Sharpe H, Xu Q, Van Nostrand WE, Vitek MP: Expression profiles for macrophage alternative activation genes in $A D$ and in mouse models of AD. J Neuroinflammation 2006, 3:27.

23. Coleman DL: Obese and diabetes: two mutant genes causing diabetesobesity syndromes in mice. Diabetologia 1978, 14:141-148.

24. Macia L, Delacre M, Abboud G, Ouk TS, Delanoye A, Verwaerde C, Saule P, Wolowczuk I: Impairment of dendritic cell functionality and steady-state number in obese mice. J Immunol 2006, 177:5997-6006.

25. Nishina PM, Lowe S, Wang J, Paigen B: Characterization of plasma lipids in genetically obese mice: the mutants obese, diabetes, fat, tubby, and lethal yellow. Metabolism 1994, 43:549-553.

26. Kleinfeld AM, Prothro D, Brown DL, Davis RC, Richieri GV, DeMaria A: Increases in serum unbound free fatty acid levels following coronary angioplasty. Am J Cardiol 1996, 78:1350-1354.

27. Shafrir E: Partition of unesterified fatty acids in normal and nephrotic syndrome serum and its effect on serum electrophoretic pattern. J Clin Invest 1958, 37:1775-1782.

28. Samokhvalov V, Bilan PJ, Schertzer JD, Antonescu CN, Klip A: Palmitate- and lipopolysaccharide-activated macrophages evoke contrasting insulin responses in muscle cells. Am J Physiol Endocrinol Metab 2009, 296:E37-E46.

29. Bunn RC, Cockrell GE, Ou Y, Thrailkill KM, Lumpkin CK Jr, Fowlkes JL: Palmitate and insulin synergistically induce IL-6 expression in human monocytes. Cardiovasc Diabetol 2010, 9:73.

30. Little JP, Madeira JM, Klegeris A: The saturated fatty acid palmitate induces human monocytic cell toxicity toward neuronal cells: exploring a possible link between obesity-related metabolic impairments and neuroinflammation. J Alzheimers Dis 2012, 30(Suppl 2):S179-S183.

31. David S, Kroner A: Repertoire of microglial and macrophage responses after spinal cord injury. Nat Rev Neurosci 2011, 12:388-399.

32. Krogh-Madsen R, Plomgaard P, Akerstrom T, Moller K, Schmitz O, Pedersen BK: Effect of short-term intralipid infusion on the immune response during low-dose endotoxemia in humans. Am J Physiol Endocrinol Metab 2008, 294:E371-E379.

\section{doi:10.1186/1742-2094-9-276}

Cite this article as: Kappe et al:: GLP-1 secretion by microglial cells and decreased CNS expression in obesity. Journal of Neuroinflammation 2012 9:276. 